\title{
Calibrating volume measurements made using the dual-field conductance catheter
}

\author{
Simon P. McGuirk ${ }^{1,2}$, David J. Barron ${ }^{1}$, Dan Ewert ${ }^{3}$, John H. Coote $^{2}$ \\ ${ }^{1}$ Department of Cardiac Surgery, Birmingham Children's Hospital, Birmingham, UK; ${ }^{2}$ School of Clinical and Experimental Medicine, \\ University of Birmingham, Birmingham, UK; ${ }^{3}$ Department of Electrical and Computer Engineering, North Dakota State University, \\ Fargo, USA. \\ Email: simon.mcguirk@,nhs.net
}

Received 1 February 2009; revised 24 June 2009; accepted 16 July 2009.

\section{ABSTRACT}

The conductance catheter technique allows realtime measurements of ventricular volume based on changes in the electrical conductance of blood within the ventricular cavity. Conductance volume measurements are corrected with a calibration coefficient, $\alpha$, in order to improve accuracy. However, conductance volume measurements are also affected by parallel conductance, which may confound calibration coefficient estimation. This study was undertaken to examine the variation in $\alpha$ using a physical model of the left ventricle without parallel conductance. Calibration coefficients were calculated as the conductance-volume quotient $\left(\alpha_{V(t)}\right)$ or the stroke conductance-stroke volume quotient $\left(\alpha_{S V}\right)$. Both calibration coefficients varied as a non-linear function of the ventricular volume. Conductance volume measurements calibrated with $\alpha_{V(t)}$ estimated ventricular volume to within $2.0 \pm 6.9 \%$. By contrast, calibration with $\alpha_{S V}$ substantially over-estimated the ventricular volume in a volume-dependent manner, increasing from $26 \pm 20 \%$ at $100 \mathrm{ml}$ to $106 \pm 36 \%$ at $500 \mathrm{ml}$. The accuracy of conductance volume measurements is affected by the choice of calibration coefficient. Using a fixed or constant calibration coefficient will result in volume measurement errors. The conductance-stroke volume quotient is associated with particularly significant and volume-dependent mea- surement errors. For this reason, conductance volume measurements should ideally be calibrated with an alternative measurement of ventricular volume.

Keywords: Conductance Catheter; Calibration; Volume Measurement

\section{INTRODUCTION}

The conductance catheter technique is an established method to measure the ventricular volume in real-time, based on the electrical conductance of blood within the ventricular cavity $[1,2,3]$. Conductance volume measurements are based on the assumption that the electric field produced by the conductance catheter is homogeneously distributed within the ventricular cavity [1]. However, theoretical and experimental studies have demonstrated that this assumption is not valid $[4,5,6]$. As a result, the conductance catheter tends to overestimate the volume in small ventricles and underestimate the volume in larger ventricles [2,3].

The dimensionless calibration coefficient, $\alpha$, was introduced by Baan et al. [3] in order to account for the non-uniform conductance-absolute volume relationship [3]. This calibration coefficient represents the slope of the relationship between the conductance-derived volume and the true volume. The calibration coefficient, $\alpha$ may also vary with ventricular volume. It is relatively high in small animals [7], lower in humans [3] and intermediate values are found in dogs $[3,8,9]$. Experimental studies demonstrate that $\alpha$ also varies during inferior vena caval occlusion [9] and may even fluctuate during the normal cardiac cycle $[10,11]$.

The tissues and fluid surrounding the ventricular cavity also contribute to the measured conductance signal [3]. This creates a volume offset called parallel conductance. Parallel conductance also varies according to the ventricular volume $[4,7,8,9,12,13]$. This volume- dependent parallel conductance may confound the calibration coefficient estimation.

This study was undertaken to examine the variation in the calibration coefficient, $\alpha$, over a range of volumes pertaining to clinical studies, in a physical model of the left ventricle without parallel conductance. 


\section{METHODS}

\subsection{Model Ventricle}

This study used a physical model of the left ventricle previously described by this laboratory [14]. This model consisted of an ellipsoid latex balloon enclosed in a pressurised Perspex chamber (Figure 1). The chamber was filled with distilled water and hydraulically pressurised with an intra-aortic balloon pump (IABP; Datascope Medical Co. Ltd, Huntingdon, UK) with a paediatric volume-limiting chamber. The IABP console was connected to two $25 \mathrm{ml}$ intra-aortic balloon catheters in parallel. The IABP circuit was filled with helium gas to ensure a rapid pneumatic response.

The electrocardiogram (ECG) from a patient simulator (Bioma Research Inc, Quebec, Canada) was used to trigger the IABP console at a predetermined rate $(60$ beats $\left.\cdot \min ^{-1}\right)$. Inflation of the IABP balloon catheters caused a rise in the ventricular pressure. This displaced saline from the latex balloon through a 2/2-way solenoid valve (Bürkert $\mathrm{GmbH}$, Ingelfingen, Germany) into a calibrated measuring cylinder at the top of the model ventricle. Deflation of the IABP balloon catheters caused ventricular pressure to fall, which allowed the latex balloon to refill. Electronic circuitry was used to control the opening and closure times of the solenoid valve in order to simulate different contraction patterns.

For the purposes of this study, the solenoid valve remained open throughout the model ventricular cycle to produce isobaric contractions. Each inflation of the IABP balloon catheters displaced a fixed stroke volume $(S V)$ of $50 \mathrm{ml}$. The stroke volume was independently

quantified before each experiment. The IABP balloon catheters were manually inflated and the displaced volume was measured in the measuring cylinder.

Three separate latex balloons were used to simulate the change in volume that may be observed during inferior vena caval occlusion. Each balloon was $13 \mathrm{~cm}$ in length and had a maximal volume at atmospheric pressure of $125 \mathrm{ml}, 215 \mathrm{ml}$ and $500 \mathrm{ml}$, respectively. The shape of the balloons was ellipsoid at low volumes and became increasingly spheroid as the volume increased. The length-diameter ratio at maximal filling decreased from 3.03 for the smallest balloon to 1.52 for largest balloon.

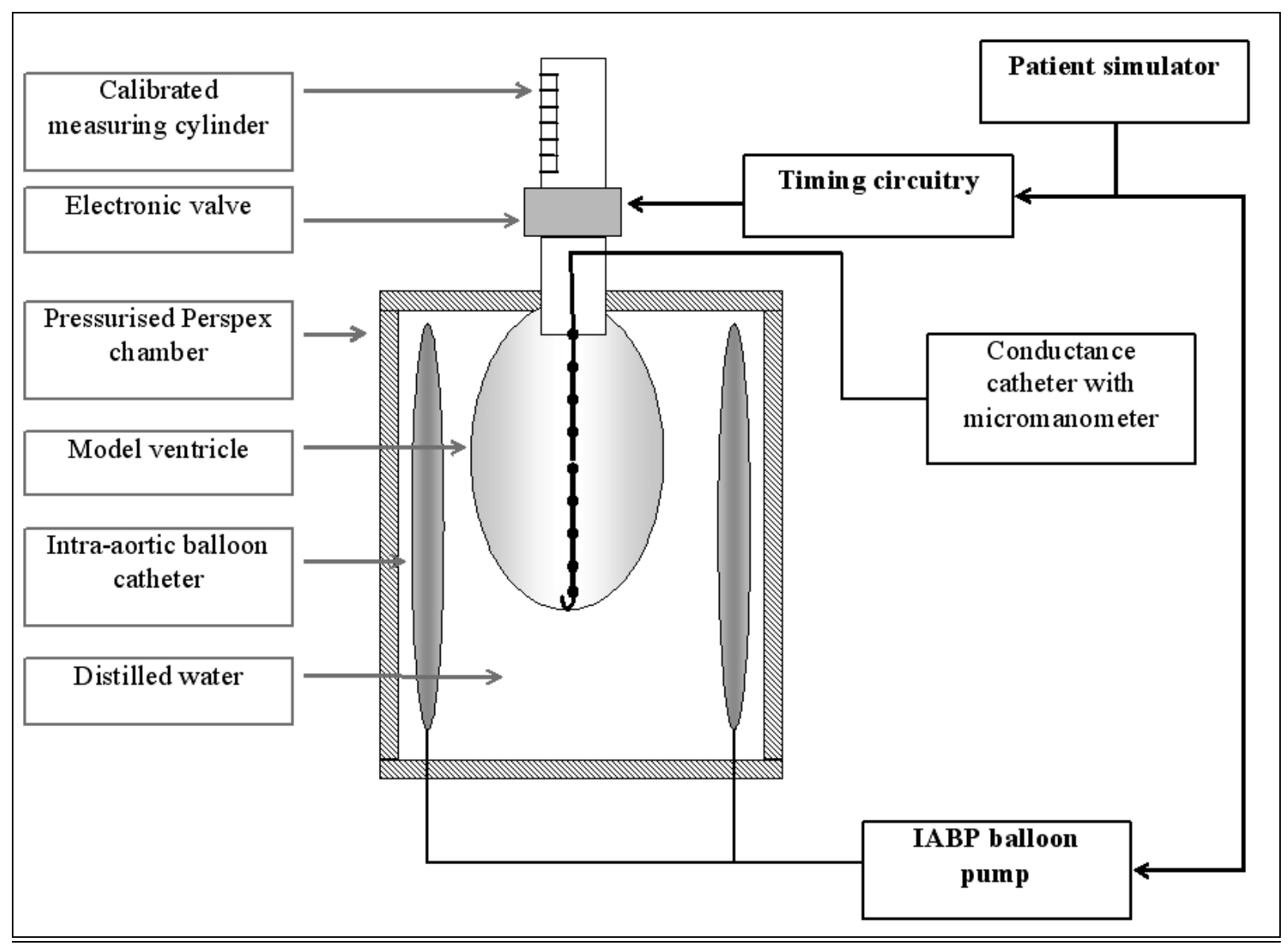

Figure 1. Schematic diagram of the model heart consisting of a model ventricle and outflow tract. The position of the conductance catheter is also illustrated. 
During each experiment, the model ventricle was filled with a known volume of $0.9 \%$ normal saline at room temperature. The end-diastolic volume $\left(V_{E D}\right)$ was varied between $100-125 \mathrm{ml}$ for the $125 \mathrm{ml}$ balloon; 160 $215 \mathrm{ml}$ for the $215 \mathrm{ml}$ balloon; and $410-500 \mathrm{ml}$ for the $500 \mathrm{ml}$ balloon. The resistivity (conductivity ${ }^{-1}$ ) of this solution was measured before each test using a dedicated measuring cuvette (CD Leycom, Zoetermeer, The Netherlands). The mean $\pm \mathrm{SD}$ resistivity was $68.3 \pm 0.5$ $\Omega \cdot \mathrm{cm}$.

\subsection{Conductance Catheter}

The principle of the conductance catheter technique for measuring LV volume has been described elsewhere [3]. In this study, a 7-French 12-electrode high-fidelity dual pressure-volume conductance catheter (Millar Instruments, Houston, TX, USA) was used. The electrodes were mounted at $10 \mathrm{~mm}$ intervals near the tip of the catheter. One alternating current $(20 \mathrm{kHz}, 30 \mu \mathrm{A}$ RMS $)$ was applied between the two outermost electrodes (electrodes 1 and 12) and a second alternating current (20 $\mathrm{kHz},-10 \mu \mathrm{A}$ RMS) was applied between the two adjacent electrodes (electrodes 2 and 11). This dual-field configuration was used in all studies [15]. A signal processor unit (CFL-512; CD Leycom) was used to measure the potential difference between seven consecutive pairs of the remaining eight electrodes (electrodes 3-10). These measured voltages were converted into seven time-varying segmental conductance signals, $G_{i}(t)$.

The conductance volume, $Q(t)$, was measured using the following formula:

$$
Q(t)=L^{2} \cdot \rho \cdot\left(\left[\sum_{i=1}^{7} G_{i}(t)\right]-G_{P}\right)
$$

where $\rho$ is the blood resistivity, $L$ is the inter-electrode distance, and $G_{P}$ is parallel conductance.

By assuming that parallel conductance is negligible (i.e. $G_{P}=0$ ), this formula can be simplified to:

$$
Q(t)=L^{2} \cdot \rho \cdot\left[\sum_{i=1}^{7} G_{i}(t)\right]
$$

The volume estimated using the conductance catheter technique was then calculated:

$$
V_{g}(t)=\frac{1}{\alpha} \cdot Q(t)
$$

where $\alpha$ is the dimensionless calibration coefficient [3].

\subsection{Experimental Data}

Analogue signals representing 7 segmental conductance volumes within the model ventricle and the ECG were digitised at 12-bit accuracy and a sample frequency of $250 \mathrm{~Hz}$. End-diastole and end-systole were retrospectively identified. End-diastole was defined as the R wave on the ECG and end-systole was defined as the point immediately prior to IABP circuit deflation.

Each experiment was conducted three times and, in each, data from 10 consecutive cycles were analysed. The estimated within-experiment standard deviation was $0.45 \mathrm{ml}$. The standard deviation was not significantly correlated with the mean conductance volume (Kendall's $\tau$ coefficient $=-0.72$ ), and so subsequent analyses were based on the average data from each experiment [16].

Two separate calibration coefficients were calculated. Firstly, the calibration coefficients, $\alpha_{V(t)}$ was calculated by dividing the conductance-derived volume measurement by the absolute ventricular volume at either end-diastole or end-systole:

$$
\alpha_{V(t)}=\frac{Q_{E D}}{V_{E D}} \text { or } \frac{Q_{E S}}{V_{E S}}
$$

where $V_{E S}=V_{E D}-S V$.

Including a "phase of measurement" (i.e. $E D$ or $E S$ ) in this equation did not improve the goodness of fit of the model. The effect of "phase of measurement" was not significant $(P=0.38)$ and was therefore not included in the model used to predict $\alpha_{V(t)}$.

The conductance-stroke volume quotient, $\alpha_{S V}$, was calculated by dividing the conductance-derived stroke volume by the absolute stroke volume:

$$
\alpha_{S V}=\frac{\left(Q_{E D}-Q_{E S}\right)}{S V}
$$

Finally, the measurement error, $\zeta$ was calculated as a percentage of the absolute ventricular volume:

$$
\zeta=\frac{V_{g}(t)}{V(t)} \cdot 100
$$

The terms $\zeta_{V(t)}$ and $\zeta_{S V}$ were used to denote the measurement error associated with $\alpha_{V(t)}$ and $\alpha_{S V}$, respectively.

\subsection{Data Analysis}

Data were analysed using SPSS for Windows (v12, SPSS Inc., Chicago, IL, USA). Conductance volumes, calibration coefficients and measurement errors were in turn examined as functions of the known volume of the model ventricle. These relationships were evaluated by least squares linear regression based on fractional polynomials of the ventricular volume. The standard error for the regression coefficients and intercept were also calculated. A probability, $P<0.05$, was taken to represent statistical significance in these analyses. Correlation coefficients between the variables were also calculated.

\section{RESULTS}

\subsection{Relationship between Conductance and Absolute Volume}

The simultaneous conductance volume, $Q(t)$, and absolute volume measurements, $V(t)$ for the three balloons 
are illustrated in Figure 2. There was a non-linear relationship between these two volume measurements. When analysed as a whole, the conductance-absolute volume relationship was best approximated by the following relationship (Eq. 7).

$$
\begin{aligned}
& Q(t)=[(14.2 \pm 0.39) \cdot \sqrt{\text { volume }}] \\
& -[(0.17 \pm 0.02) \cdot \text { volume }],\left(r^{2}=0.90, P<0.001\right)
\end{aligned}
$$

Compared with the line of identity (i.e. $x=y$ ), conductance volume measurements predicted using this model were equal to ventricular volume at approximately $150 \mathrm{ml}$; slightly overestimated ventricular volume when the absolute volume was less than $150 \mathrm{ml}$; but underestimated ventricular volume at volumes over 150 $\mathrm{ml}$.

The volume measurements formed two distinct subsets. Data from the small and medium balloons were clustered on the left-hand side of the plot while data from the large balloon was clustered on the right-hand side. When considered individually, the conductance-absolute volume relationship was well approximated by linear regression (Eq.s 8 and 9). In neither case was the quadratic term significant. For the small and medium-sized balloons, the linear regression was:

$$
\begin{aligned}
& Q(t)=[(0.63 \pm 0.06) \cdot V(t)]+(55 \pm 9) \\
& \left(r^{2}=0.90, P<0.001\right)
\end{aligned}
$$

while for the large balloon, the corresponding equation was:

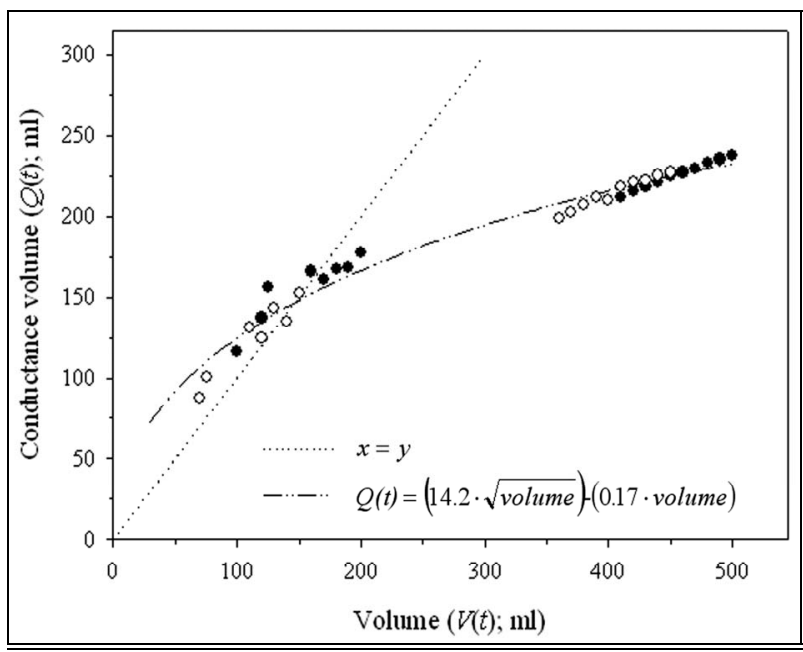

Figure 2. Conductance volume measurements, $Q(t)$ versus absolute volume, $V(t)$ at end-diastole $(\bullet)$ and end-systole $(\circ)$ for each of the three latex balloons. The curve (dashed line) represents the regression analysis of the conductance-absolute volume relationship. The curve (dotted line) representing the line of identity $(x=y)$ is also illustrated.

$$
\begin{aligned}
& Q(t)=[(0.27 \pm 0.01) \cdot V(t)]+(104 \pm 5), \\
& \left(r^{2}=0.96, P<0.001\right)
\end{aligned}
$$

The slope of these two mathematical models are significantly different from one another $(P<0.05)$, further illustrating that a single, common linear relationship does not hold over the entire range of absolute volumes. In addition, the intercepts differ significantly from one another and from zero $(P<0.05)$. Therefore, neither of these two lines passes through the origin. This introduces a "volume offset", which in this apparatus cannot be due to parallel conductance.

\subsection{Relationship between Calibration Coefficients and Absolute Volume}

The calibration coefficients, $\alpha_{V(t)}$ and $\alpha_{\mathrm{SV}}$ were calculated using Eq.s 4 and 5, respectively. Neither calibration coefficient was constant or linearly related to the ventricular volume. Instead, both calibration coefficients decreased progressively as the ventricular volume increased (Figures 3A and 3B). The calibration coefficient, $\alpha_{V(t)}$ varied as a function of the square root of the absolute volume $\left(\mathrm{r}^{2}=0.97, P<0.001\right)$. By contrast, $\alpha_{\mathrm{SV}}$ varied as a function of the inverse absolute volume $\left(\mathrm{r}^{2}=0.98\right.$, $P<0.001$; and $\mathrm{r}^{2}=0.93, P<0.001$ for end-diastolic and end-systolic measurements respectively).

The relative ratio of the two calibration coefficients (i.e. $\left.\alpha_{S V} / \alpha_{V(t)}\right)$ was also examined over the volume range (Figure 3C). The stroke volume quotient, $\alpha_{S V}$ was lower than $\alpha_{V(t)}$ at each volume measurement. However, the slope calibration coefficient ratio was not constant but became progressively smaller as the absolute volume was increased. For example, the $\alpha_{S V} / \alpha_{V(t)}$ ratio decreased from $0.98 \pm 0.52$ to $0.43 \pm 0.23$ as the end-diastolic volume increased from $100 \mathrm{ml}$ to $500 \mathrm{ml}$.The best approximation for this relationship was that the $\alpha_{S V} / \alpha_{V(t)}$ ratio varied as a function of the inverse ventricular volume $\left(\mathrm{r}^{2}=0.81, P<0.001 ; \mathrm{r}^{2}=0.75, P<0.001\right.$ for end-diastolic and end-systolic measurements, respectively).

\subsection{Relationship between Calibrated Conductance and Absolute Volume and Measurement Error}

Calibrated conductance volume measurements, $V_{g}(t)$ were calculated using Eq. 3, in which $\alpha$ was either $\alpha_{V(t)}$-volume or $\alpha_{S V}$-volume relationship, as previously described (Figure 4). Conductance volume measurements calibrated with the $\alpha_{V(t)}$-volume relation increased linearly with the absolute volume $\left(\mathrm{r}^{2}=0.99, P<0.001\right)$. Conductance volume measurements calibrated with the $\alpha_{S V}$-volume relation also increased linearly with absolute volume $\left(\mathrm{r}^{2}=0.99, P<0.001\right)$, but overestimated the absolute volume. 


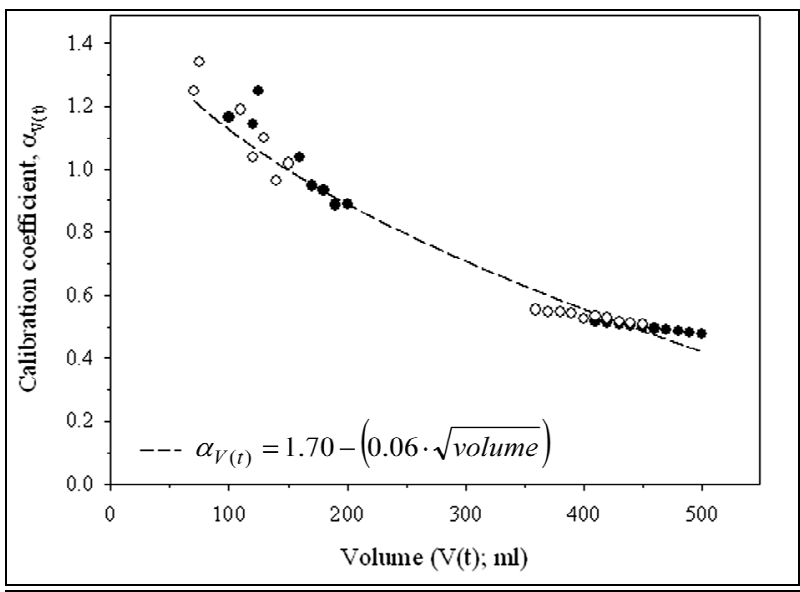

(a)

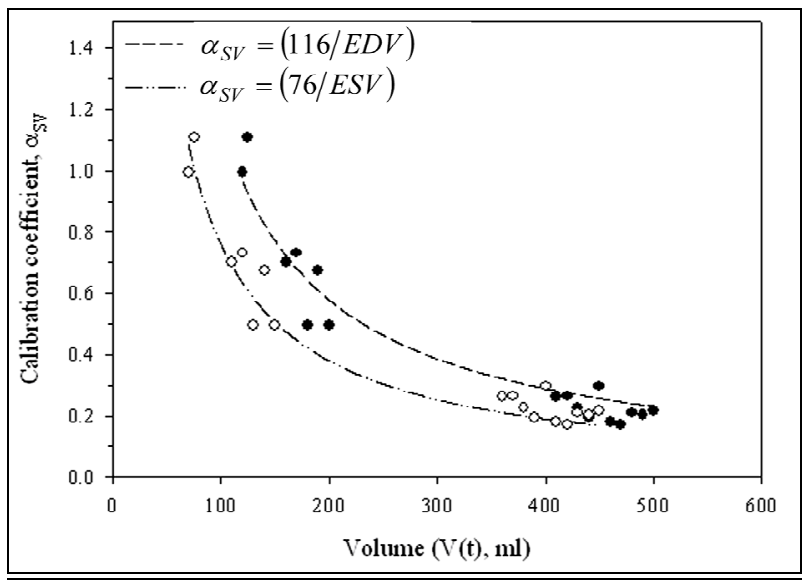

(b)

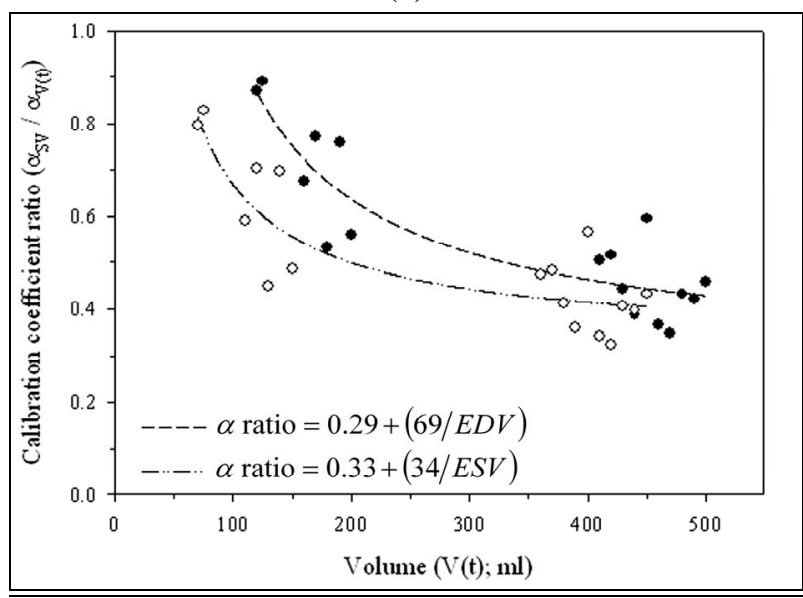

(c)

Figure 3. Calibration coefficient, $\alpha$ versus absolute volume, $V(t)$ at end-diastole $(\bullet)$ and end-systole $(\circ)$ for each of the three latex balloons. The calibration coefficient was calculated as the conductance-absolute volume quotient $\left(\alpha_{V(t) ;} \mathrm{A}\right)$ or the conductance-stroke volume quotient $\left(\alpha_{S V}, \mathrm{~B}\right)$. The calibration coefficient ratio (C) represented $\alpha_{S V}$ as a proportion of $\alpha_{V(t)}$. Curves (dashed lines) representing regression analyses of the calibration coefficient-conductance volume relations are also illustrated.
The volume measurement error, expressed as a percentage of the absolute volume, was calculated for each calibration coefficient (Figure 5). Conductance volume measurements calibrated with the $\alpha_{V(t)}$-volume relation had a measurement error of $2.0 \pm 6.9 \%$, which was relatively constant across the span of volume measurements. On the other hand, the measurement error associated with the $\alpha_{S V}$-volume relation was volume-dependent, increasing linearly from $26 \pm 20 \%$ to $106 \pm 36 \%$ as the end-diastolic volume increased from $100 \mathrm{ml}$ to $500 \mathrm{ml}$ $\left(\mathrm{r}^{2}=0.96, P<0.001\right)$.

\section{DISCUSSION}

The non-homogeneous electric field generated by the conductance catheter results in a non-linear relationship between conductance and true ventricular volume measurements. Conductance measurements are, therefore, calibrated using the dimensionless calibration coefficient, $\alpha$ in order to obtain absolute volume measurements. This study investigated the accuracy of conductance volume measurements, and the effect of calibration, in a series of in vitro experiments that spanned the volume range observed in clinical studies [17]. This model design also avoided the potential problems associated with parallel conductance.

This study has confirmed that there is a non-linear relationship between dual-field conductance and absolute volume measurements, such that the conductance- volume relation is concave towards the true volume. The conductance volume measurements underestimated ventricular volume as the volume was increased above $150 \mathrm{ml}$. This finding is in accord with previous experimental studies $[4,6]$.

Conductance volume measurements are generally calibrated with the dimensionless calibration coefficient in order to improve the accuracy of ventricular volume measurements made using this technique [3]. In the isolated post-mortem canine heart, Mur \& Baan reported that the conductance-volume relation was virtually linear over a finite volume range [2] and the authors predicted that a similar, virtually linear conductance-volume relation would be observed for the human left ventricle up to a volume of $200 \mathrm{ml}$ [2].

However, this study has shown that the calibration coefficient, $\alpha$ is not constant, but varies as a non-linear function of the ventricular volume. Using a fixed or constant $\alpha$ during acute volume change will inevitably result in volume measurement errors. These measurement errors may be relatively small during the normal cardiac cycle. However, procedures that produce an acute and substantial change in volume load, like vena caval occlusion, will potentially result in significant measurement errors.

Kornet et al. suggested that the variation in $\alpha$ observed in vivo might reflect synchronous volume-dependent 


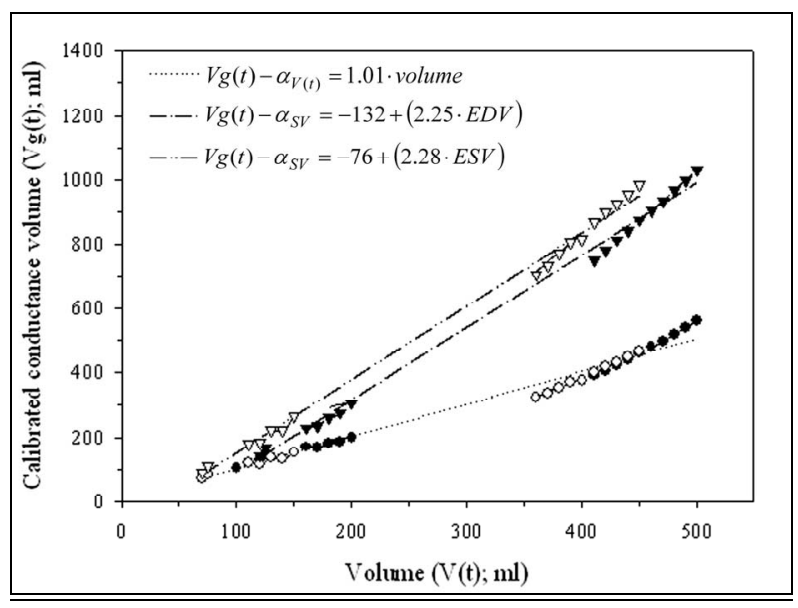

Figure 4. Calibrated conductance volume measurements, $V_{g}(t)$ versus absolute volume, $V(t)$ at end-diastole $(\bullet)$ and end-systole $(\circ)$ for each of the three latex balloons. Conductance volume measurements were calibrated using either $\alpha_{V(t)}$ $(\bullet, \circ)$ or $\alpha_{S V}(\nabla, \nabla)$.Curves (dashed lines) representing regression analyses of the calibrated conductance volume-absolute volume relations are also illustrated.

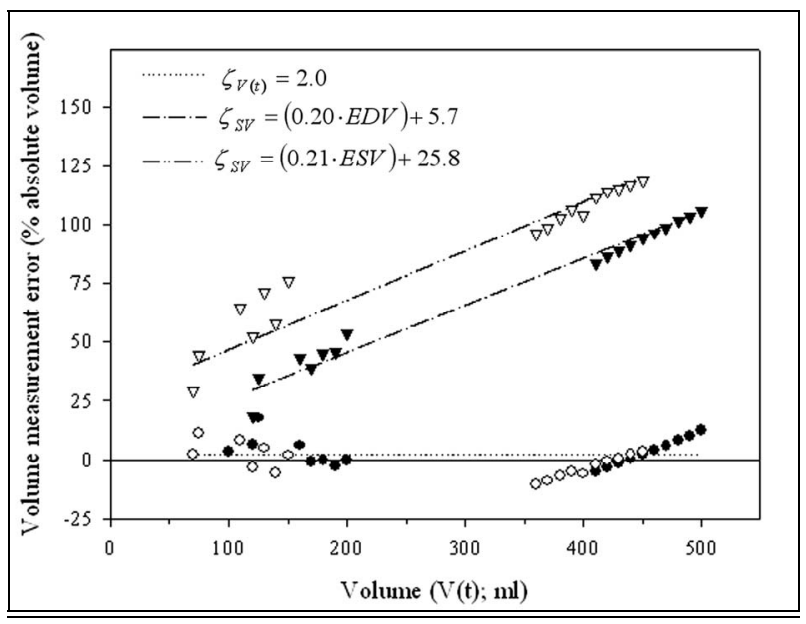

Figure 5. Calibrated conductance volume measurement error, $\zeta$ versus absolute volume, $V(t)$ at end-diastole $(\bullet)$ and end-systole ( $\circ$ ).Conductance volume measurements calibrated using either $\alpha_{V(t)}(\bullet, \circ)$ or $\alpha_{S V}(\boldsymbol{\nabla}, \nabla)$. Volume measurement error was calculated as a percentage of the absolute volume. Curves (dotted and dashed lines) representing regression analyses of the measurement error-absolute volume relations are also illustrated.

changes in parallel conductance during the cardiac cycle [13]. However, this study used a physical model of the left ventricle without parallel conductance. Our findings suggest that parallel conductance is not a comprehensive explanation for why the calibration coefficient varies as a function of the ventricular volume.

Although conductance volume measurements can be calibrated against another synchronous estimate of ventricular volume $[3,10,18]$, they are generally calibrated with an alternate measurement of stroke volume $[6,10]$.
Calibration with either an alternate measurement of ventricular volume or stroke volume are generally considered equivalent. This study also identified that the $\alpha_{V(t)}$ and $\alpha_{S V}$ are different. The stroke volume quotient, $\alpha_{S V}$ was lower than $\alpha_{V(t)}$ and the calibration coefficient ratio, $\alpha_{S V} / \alpha_{V(t)}$ became progressively smaller as the ventricular volume increased. Calibrating conductance volume measurements with $\alpha_{S V}$ resulted in the significant overestimation of end-diastolic and end-systolic volume, and the degree of overestimation was even more pronounced at higher volumes. This demonstrates that conductance volume measurements should ideally be calibrating using an independent measure of ventricular volume like, for example, contrast cineangiography, echocardiography or magnetic resonance imaging.

The difference between the $\alpha_{V(t)}$-volume and $\alpha_{S V^{-}}$ volume relations may be attributed to the non-homogeneous electrical field distribution established by the conductance catheter $[4,12]$. Conductance volume measurements are disproportionately influenced by the areas around the longitudinal axis of the ventricle where the electrical field is strongest. By contrast, changes in volume that occur during ejection or when loading conditions are varied primarily affect the myocardial boundary where the electrical field density is weakest. Consequently, $\alpha_{S V}$ and $\alpha_{V(t)}$ are not equivalent and cannot be used interchangeably.

\subsection{Study Limitations}

The physical model of the isolated left ventricle used in this study did not allow changes in the volume loading conditions could not be modelled continuously. Instead, a series of steady-state experiments were made at incremental end-diastolic volumes in three separate models, which carries with it the risk of repeated measurement errors. The changes in ventricular volume resulted from changes in the short-axis dimension of the model only. It was not possible to examine any effect of changing ventricular length, such as those that may occur during the cardiac cycle [19].

Ideally, the volumes within the model ventricle would have been acquired simultaneously using the conductance catheter and another independent method. In the original design, the ejected volume was measured according to the pressure generated within the fluid column [14]. However, preliminary experiments demonstrated that the fluid-filled micromanometer was not sufficiently accurate to enable instantaneous volume measurements throughout the cardiac cycle and, therefore, measurements were made with the calibrated measuring cylinder.

\section{CONCLUSIONS}

The conductance catheter technique incorporates a calibration coefficient, $\alpha$, in order to obtain accurate volume 
measurements. This study has demonstrated that this calibration coefficient varies as a function of absolute volume, independent of parallel conductance. Assuming that the calibration coefficient is fixed or constant will introduce measurement errors. The conductance-stroke volume quotient, $\alpha_{S V}$ is associated with particularly significant and volume-dependent measurement errors. This limits the value of volume measurements calibrated using $\alpha_{S V}$. Conductance volume measurements should ideally be calibrated with an alternative measurement of ventricular volume, using any one of the techniques that are now available.

\section{ACKNOWLEDGEMENTS}

Simon McGuirk was supported by a British Heart Foundation Junior Research Fellowship (FS/03/102).

\section{REFERENCES}

[1] Baan, J., Aouw Jong, T. T., Kerkhof, P. L., Moene, R. J., van Dijk, A. D., van der Velde, E. T., and Koops, J., (1981) Continuous stroke volume and cardiac output from intra-ventricular dimensions obtained with an impedance catheter. Cardiovascular Research, 15, 328-334.

[2] Mur, G. and Baan, J., (1984) Computation of the input impedances of a catheter for cardiac volumetry, IEEE Transactions on Biomedical Engineering, 31, 448-453.

[3] Baan, J., van der Velde, E. T., de Bruin, H. G., Smeenk, G. J., Koops, J., van Dijk, A. D., Temmerman, D., Senden, J. and Buis, B., (1984) Continuous measurement of left ventricular volume in animals and humans by conductance catheter, Circulation, 70, 812-823.

[4] Wu, C. C., Skalak, T. C., Schwenk, T. R., Mahler, C. M., Anne, A., Finnerty, P. W., Haber, H. L., Weikle II, R. M. and Feldman, M. D., (1997) Accuracy of the conductance catheter for measurement of ventricular volumes seen clinically: Effects of electric field homogeneity and parallel conductance, IEEE Transactions on Biomedical Engineering, 44, 266-277.

[5] Salo, R. W., (1992) Improvements in intracardiac impedance volumes by field extrapolation, European Heart Journal, 13(Suppl E), 35-39.

[6] Wei, C. L., Valvano, J. W., Feldman, M. D. and Pearce, J. A., (2005) Nonlinear conductance-volume relationship for murine conductance catheter measurement system. IEEE Transactions on Biomedical Engineering, 52, 654661.

[7] Cassidy, S. C. and Teitel, D. F., (1992) The conductance volume catheter technique for measurement of left ventricular volume in young piglets, Pediatric Research, 31,
85-90.

[8] Boltwood, C. M., Appleyard, R. F., and Glantz, S. A. (1989) Left ventricular volume measurement by conductance catheter in intact dogs: parallel conductance volume depends on left ventricular size, Circulation, 80, 1360-1377.

[9] Applegate, R. J., Cheng, C. P. and Little, W. C., (1990) Simultaneous conductance catheter and dimension assessment of left ventricular volume in the intact animal, Circulation, 81, 638-648.

[10] Szwarc, R. S., Laurent, D., Allegrini, P. R., and Ball, H. A., (1995) Conductance catheter measurement of left ventricular volume; evidence for nonlinearity within cardiac cycle, American Journal of Physiology-Heart and Circulatory Physiology, 268, H1490-H1498.

[11] Danton, M.H., Greil, G.F., Byrne, J.G., Hsin, M. Cohn, L. and Maier, S.E. (2003) Right ventricular volume measurement by conductance catheter. American Journal of Physiology-Heart and Circulatory Physiology, 285, H1774-H1785.

[12] Kun, S. and Peura, R. A., (1994) Analysis of conductance volumetric measurement error sources, Medical and Biological Engineering and Computing, 32, 94-100.

[13] Kornet, L., Schreuder, J. J., van der Velde, E. T., and Jansen, J. R., (2001) The volume-dependency of parallel conductance throughout the cardiac cycle and its consequence for volume estimation of the left ventricle in patients, Cardiovascular Research, 51, 729-735.

[14] Al-Khalidi, A. H., Townend, J. N., Bonser, R. S., and Coote, J. H., (1998) Validation of the conductance catheter method for measurement of ventricular volumes under varying conditions relevant to cardiac surgery, American Journal of Cardiology, 82, 1248-1252.

[15] Steendijk, P., van der Velde, E. T., and Baan, J., (1992) Single and dual excitation of the conductance-volume catheter analysed in a spheroidal mathematical model of the canine left ventricle, European Heart Journal, 13 (Suppl E), 28-34.

[16] Bland, J. M. and Altman, D. G., (1996) Statistics notes: Measurement error, British Medical Journal, 313, 744.

[17] Tkacova, R., Hall, M. J., Liu, P. P., Fitzgerald, F. S., and Bradley, T. D., (1997) Left ventricular volume in patients with heart failure and Cheyne-Stokes respiration during sleep, American Journal of Respiratory Care Medicine, 156, 1549-1555.

[18] Kass, D. A., (1992) Clinical evaluation of left heart function by conductance catheter technique, European Heart Journal, 13(Suppl E), 57-64.

[19] Rushmer, R. F., Crystal, D. K., and Wagner, C., (1953) The functional anatomy of ventricular contraction, Circulation Research, 1, 162-70. 